\title{
Does intraoperative application of TachoSil reduce the number of lymphoceles after pelvic lymphadenectomy?
}

\author{
Andrzej Wrobel ${ }^{1}{ }^{\mathbb{D}}$, Izabela Winkler ${ }^{2}$, Tomasz Rechberger ${ }^{1}{ }^{\mathbb{D}}$, Katarzyna Skorupska ${ }^{1} \mathbb{D}$, Pawel Miotla $^{1}$ \\ ${ }^{1} 2^{\text {nd }}$ Department of Gynecology, Medical University of Lublin, Poland \\ ${ }^{2} 2^{\text {nd }}$ Department of Gynecology, St' Johns Centre Oncology, Lublin, Poland
}

\begin{abstract}
Objectives: The degree of lymphoceles prevention was assessed using collagen patches coated with human coagulation factors (TachoSil, Nycomed International Management GmbH, Zurich, Switzerland). The study enrolled 50 consecutive patients with endometrial and cervical cancer stages IB to II who had undergone open hysterectomy and pelvic lymphadenectomy (PL). In addition, the drainage volumes of 22 patients with hypertension were compared to that of the rest of the study population. Furthermore, occurrence of lymphocele in patients with endometrial and cervical cancer were compared after completion of adjuvant treatment.
\end{abstract}

Material and methods: Patients were simultaneously randomized in two groups: as a control (side without TachoSil applied) and study group (side with TachoSil applied). All surgical parameters were collected, and patients underwent ultrasound examination on postoperative days 1,6 , and 30 , and at the end of treatment.

Results: The TachoSil Group showed a lower drainage volume, 30 days after surgery, while outflow of fluid occurred in 11 (22\%) of all TachoSil Group cases and 22 (44\%) of all control group cases. Furthermore, two patients in the control group had symptomatic lymphocele, while the same number of cases was observed in the TachoSil Group. However, the TachoSil Group demonstrated a decreased tendency to lymphocele occurrence after the end of adjuvant therapy. Here, patients with the collagen patch developed lymphocele in $12 \%$ of all cases, as opposed to $18 \%$ without TachoSil.

Conclusions: TachoSil is a useful support treatment option for reducing drainage volume and preventing lymphocele development after lymphadenectomy.

Key words: Iymphocele; endometrial cancer; lymphadenectomy; cervical cancer; collagen patch

\section{INTRODUCTION}

Lymphoceles are the most common postoperative complications of pelvic lymphadenectomy among patients with endometrial and cervical cancer [1]. Incidences of lymphocele occur in $1 \%$ to $50 \%$ of all cases and depend on the surgical technique used and the extent of lymphadenectomy [2]. Symptoms are pelvic pain, leg edema, gastrointestinal obstruction, obstructive uropathy, and deep vein thrombosis, sepsis and lymphatic fistula formation. Easy migration of lymph from lymphatic vessels into tissues results from low concentration of coagulation factors, absence of thrombocytes and lack of smooth muscle in the lymph vessel walls. All these factors prevent lymph vessel walls from shrinking. There is also an accumulation of high-protein interstitial fluid with a low oxygen content and proteolytic activity that results in a chronic inflammation. Lymphoceles are clinically significant as they increase the cost of treatment and they prolong hospitalization, reduce the quality of patients' life, increase morbidity and, what is very important, delay the onset of adjuvant oncological treatment. Due to the lack of methods of intraoperative diagnosis of damage in the lymphatic system and the ineffectiveness of thermal ablation in relation to the closure of lymphatic vessels, there is a great need to develop methods for effective lymphocele prophylaxis.

\footnotetext{
Corresponding author:

Katarzyna Skorupska

$2^{\text {nd }}$ Department of Gynecology, Medical University of Lublin, 8 Jaczewskiego St, 20-954 Lublin, Poland

e-mail: kasiaperzylo@hotmail.com
} 
Several techniques have been described to decrease the incidence of lymphocele. Some authors suggest that the peritoneum should be left open over the lymphadenectomy sites at the end of the procedure and drains should not be placed. Moreover, omentoplasty should be encouraged. Others suggest that postoperative octreotide therapy seems beneficial, but the role of this drug in pelvic oncological surgery remains to be determined [3]. Hence, further studies are needed to assess the potential benefits of new practices.

TachoSil is a fibrin-collagen patch coated with human coagulation factors and is intended to support surgical hemostatic interventions. The specific, hexagonal, multimodal structure of the collagen sponge covered with a coating of human fibrinogen and thrombin provides more than double the adhesion strength in comparison to standard tissue adhesion [4]. TachoSil contains human fibrinogen and thrombin in the form of a dry coating located on the surface of the collagen sponge. On contact with a wound, the fibrinogen is transformed into fibrin monomers that spontaneously polymerize to the fibrin clot. This effect is responsible for the adhesion of the collagen sponge to the wound surface and the sealing of the lymphatic vessels. The use of TachoSil in the prevention of lymphocele can thus be justified via its unique adhesive properties. The efficacy and safety of TachoSil has been demonstrated in gynecological oncology in the prevention of lymphocele [1, 5-7].

\section{Objectives}

The aim of the study was to evaluate the effectiveness of TachoSil in the prevention of lymphocele after the performance of lymphadenectomy in patients diagnosed with endometrial and cervical cancer. The primary endpoints were the incidence and volume of lymphocele. The period of hospitalization, duration of lymphatic drainage and the period of implementation of adjuvant treatment were secondary endpoints.

\section{MATERIAL AND METHODS}

The study was approved by the Bioethics Committee of the Medical University of Lublin, Lublin, Poland (KE-0254/276/2013). ClinicalTrials.gov ID of study is NCT03752606 from 21 November 2018.

The study population consisted of 50 women who had undergone pelvic lymphadenectomy for either cervical or endometrial cancer and who had met the inclusion/exclusion criteria requirements (Tab. 1). Inclusion criteria for the study included women undergoing open hysterectomy and lymphadenectomy for cervical or endometrial cancer, age between 18 and 70 years, and the signing of a written informed consent (patients had to agree to participate in the study and had to signal their informed consent at least one day before surgery). Exclusion criteria included

\begin{tabular}{|l|l|}
\hline \multicolumn{2}{|l|}{ Table 1. Demographics } \\
\hline Characteristics & Patients $(\mathbf{n}=\mathbf{5 0})$ \\
\hline Age (Mean, years) & $56 \pm 9.5$ \\
\hline BMI (Mean \pm SD, $\left.\mathrm{kg} / \mathrm{m}^{2}\right)$ & $26.84 \pm 4.4$ \\
\hline $\begin{array}{l}\text { Type of cancer } \\
\text { Cervical } \\
\text { Endometrial }\end{array}$ & 4 \\
\hline Operations time & 46 \\
\hline Hypertension $(\mathrm{n})$ & $89.4 \pm 60 \mathrm{~min}$ \\
\hline
\end{tabular}

$\mathrm{BMI}$ - body mass index; SD — standard deviation

women with previously diagnosed lymph edema or disease of the lymphatic system or a known disease of the immune system.

The prospective randomized clinical intervention trial consisted of 50 open surgeries undertaken between 2013-2014 at the $2^{\text {nd }}$ Department of Gynecology in Lublin. The participants were centrally randomized by the principal investigator (TR). The case surgeons allocated TachoSil (NCT Number: ICMJE NCT01192022; Other Study ID Numbers: ICMJETC-2402-040-SP; U1111-1130-9121 Registry Identifier: WHO) for one side (left or right) after lymphadenectomy. The second side was a control side without TachoSil. Allocation was communicated by telephone after informed consent had been obtained and after lymphadenectomy had been completed. Outcome assessment was performed by independent reviewers who were blinded to the treatment allocation.

The open surgeries were performed as follows: in women who underwent routine pelvic lymphadenectomy, lymph node tissue was removed from the external iliac vessels, the obturator fossa, the interiliac region, and the common iliac region after identification and appropriate preparation of iliac vessels and obturator nerve. The same amount of lymph node tissue was removed from both sides. At the end of the procedure, hemostasis was checked. A TachoSil patch of $4.8 \times 4.8 \mathrm{~cm}$ was attached to one side of the obturator fossa (study group). The same patient also constituted the control group, as no TachoSil patch was used on the second side of the lymphadenectomy. Specific drainage of the retroperitoneum was performed.

Taking into consideration the examined group that the patient was allocated to, the surgeon applied either one TachoSil patch in the study group or no TachoSil in the control group. On TachoSil placement, after four minutes, a uniform pressure was applied to it to provide rapid haemostasis by forming a strong fibrin clot adjacent to the tissue surface. The TachoSil patch was placed alternately once in the left, once in the right obturator fossa, so that each of the patients participating in the study could be their own control. The next step was a radical hysterectomy — in case 
of cervical cancer, and simple hysterectomy - in case of endometrial cancer with adnexa.

After completion of the procedure, the stitching of the vagina to the hollow was performed by passing the vaginal seam through the vaginal wall, the right-side sacro-uterus ligament, the right round ligament, the peritoneal uterine vesiculitis, the left round ligament, the left sacro-uterine ligament and, finally, the vaginal wall. This way of fixation allowed free lymphatic drainage of the retroperitoneal space. Two drains from the vicinity of the pits of the curtains were removed through the abdominal wall, which were left to the second day after surgery or longer if the volume of secretion in the drainage exceeded $40 \mathrm{~mL}$ per day. The urinary bladder catheter was removed on the third day after surgery and ultrasound after voiding (PVR) was evaluated.

Surgical procedures were performed by four doctors with extensive experience in oncological gynecology. The surgical protocols were blinded to other researchers who controlled patients in the postoperative period, and the data obtained were then analyzed by an independent reviewer. The evaluation criteria of the study were the development of lymph cysts and their volume. Antibiotic prophylaxis was implemented according to local hospital recommendations. In addition, metronidazole was administered at a dose of $500 \mathrm{mg}$ every 8 hours. i.v. for the first three days after the procedure. Patients also received small-molecule heparin at a dose of 4,000 IU from the day preceding the surgery up to 30 days after its completion. Due to lymphatic cysts usually appearing 7 to 15 days after lymphadenectomy, the ultrasound examination of the presence and volume of lymphocele was performed on the 7th and 30th day after the surgery and after completing the oncological complementary treatment [1, 8]. The criteria proposed by Tinelli et al. [1], were used to define lymphocele. Histopathological report revealed that there was $8.3 \pm 1.4 \mathrm{lymph}$ nodes removed from right side and $8.5 \pm 1.9$ from left side $(p=0.56)$.

\section{Statistical analysis}

The chi-square test was used for comparisons of frequencies and cross-tabulations. One Way ANOVA was exploited to generate ranking means. Standard statistical operations (means, standard deviations, and ranges) were employed for describing demographic data. Bonferroni's correction was applied for multiple comparisons of secondary outcomes. There was no normal distribution, therefore, a non-parametric Mann-Whitney-U test was used for statistical comparison. The Wilcoxon-paired test (Z) was enlisted to compare related samples. $\mathrm{P}$ less than 0.05 was considered significant.

\section{RESULTS \\ Demographic data}

The mean age of patients was $56 \pm 9.5$ years old, and median stay in the hospital was $11.8 \pm 8$ days, however, seven patients stayed more than 15 days. One day after surgery, outflow of clear fluid was observed in 39 (78\%) TachoSil Group cases and 40 control group cases (80\%). Two days after surgery, the same number of cases showed outflow of fluid (36 or $72 \%$ ). After six days, outflow of fluid was observed in 16 (32\%) TachoSil Group cases and 13 (26\%) control group cases. Thirty days post-operation, outflow of fluid occurred in 11 (22\%) TachoSil Group cases and 22 (44\%) control group cases. Finally, two patients of the control group had symptomatic lymphocele, while the same number of cases was observed in the TachoSil Group (Tab. 2).

\section{Estimation of the drainage volume with TachoSil application}

Figure 1 presents the drainage volume $(\mathrm{mL})$ with TachoSil applied. We found a significantly difference in drainage vol-

\begin{tabular}{|c|c|c|c|c|}
\hline \multirow{2}{*}{ Day after surgery } & \multicolumn{2}{|c|}{ TachoSil } & \multicolumn{2}{|c|}{ Control } \\
\hline & n & $\%$ & n & $\%$ \\
\hline Day 1 & 39 & 78.0 & 40 & 80.0 \\
\hline Day 2 & 36 & 72.0 & 36 & 72.0 \\
\hline Day 6 & 16 & 32.0 & 13 & 26.0 \\
\hline Day 30 & 11 & 22.0 & 22 & 44.0 \\
\hline
\end{tabular}

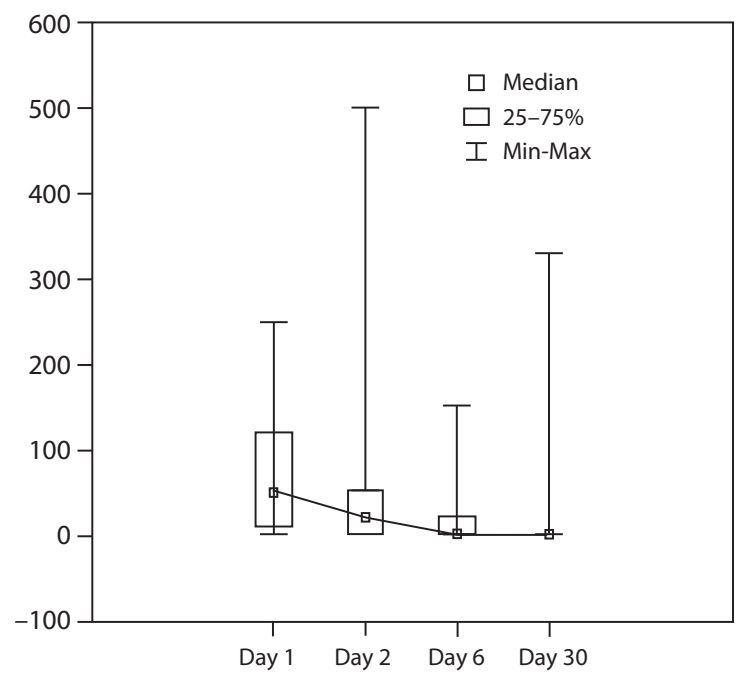

Figure 1. Amount of daily drainage - with TachoSil ( $\mathrm{mL})$ 
ume at day $1(73.56 \pm 73.03 \mathrm{~mL})$ and day $2(44.30 \pm 80.25 \mathrm{~mL}$; $\mathrm{p}=0.004)$. Moreover, we observed statistical difference between drainage volume at day $2(44.30 \pm 80.25 \mathrm{~mL})$ and day $6(14.68 \pm 31.61 \mathrm{~mL})$, respectively.

\section{Estimation of the drainage volume without TachoSil application}

Figure 2 presents the drainage volume $(\mathrm{mL})$ without TachoSil applied. We found a significant difference in drainage volume at day $2(46.8 \pm 51.05 \mathrm{~mL})$ and day $6(20.4 \pm 47.54 \mathrm{~mL}$; $\mathrm{p}<0.001)$. However, we observed no statistical difference between day $1(54.2 \pm 60.07 \mathrm{~mL})$ and $2(46.8 \pm 51.05 \mathrm{~mL})$ and between day $6(20.4 \pm 47.54 \mathrm{~mL})$ and day $30(41.52 \pm 102.07 \mathrm{~mL})$.

\section{Comparison drainage volume between groups with and without TachoSil}

Figure 3 presents the comparison drainage volume $(\mathrm{mL})$ of the groups with and without TachoSil. We found a significantly difference in drainage volume at day $30(Z=2.565$; $p=0.01)$. Here, drainage volume with TachoSil application was lower $(\mathrm{M}=14.84 \mathrm{~mL})$ in comparison to the control group (without TachoSil) $(\mathrm{M}=41.52 \mathrm{~mL})$. In contrast, we observed no statistical difference between days 1, 2 or 6 .

\section{Comparison of drainage volume between group with and without hypertension}

In comparing the 22 individuals known to have hypertension to the rest of the study population, we found no significant difference in drainage volume at day 1, 2, 6 and 30 between cases with and without hypertension.

\section{Comparison of occurrence of lymphocele in patients with endometrial and cervical cancer after completing of adjuvant treatment}

Lymphocele was observed in $44 \%$ of all control group cases and $22 \%$ of all TachoSil Group cases 30 days post-surgery. During the first-year follow-up, after completion of adjuvant therapy, we observed incidence of lymphocele decline to $18 \%$ in general and $12 \%$ in the side in which a TachoSil patch was applied (Fig. 4).

\section{DISCUSSION}

Most cases of lymphocele are found accidentally during routine medical check-ups. Here, small cysts are often asymptomatic and do not require treatment because they are self-absorbed. Indeed, in the group of patients with clinically symptomatic lymphocele, complaints associated with the presence of lymphocele normally result from the mechanism of compression of the surrounding anatomical structures and depend on the location and size of the lesions $[1,2,7]$. Song et al. [9], indicate that there is a correlation between the number of removed lymph nodes and the

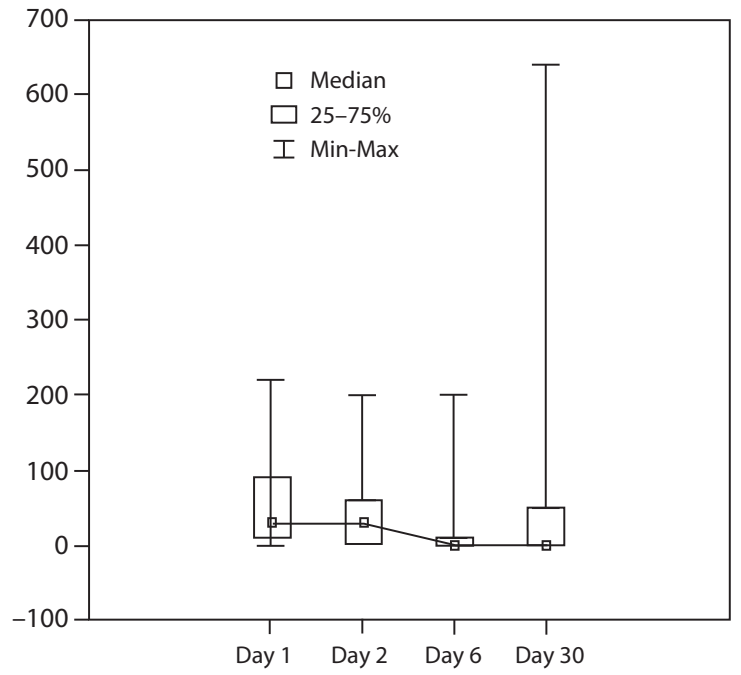

Figure 2. Amount of daily drainage - without TachoSil (mL)

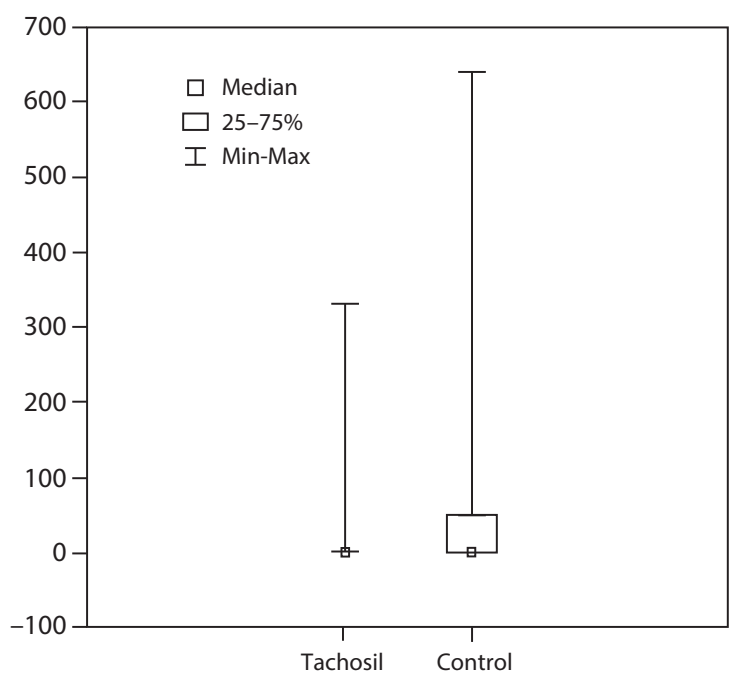

Figure 3. Comparison of drainage volume $(\mathrm{mL})$ between groups with and without Tachosil

incidence of lymphocele [9], with the risk of being higher in patients with more lymph nodes removed (37.3 vs 29.2; $p<0.02$ ). Tinelli et al. [1], and Achouri et al. [10], add that the possibility of lymphocele diagnosis should be considered after lymphadenectomy in gynecological malignancy, especially when BMI is higher than $25 \mathrm{~kg} / \mathrm{m}^{2}$, a broad lymphadenectomy has been undertaken, metastatic lymph nodes have been indicated, the patient is receiving heparin prophylaxis, lymphatic ligation failure has occurred, open surgery versus laparoscopy was applied, chemotherapy and radiotherapy has been administered.

$\mathrm{Xu}$ et al. [11], described that in women with cervical cancer, asymptomatic lymphoceles detected by ultrasound have been noted in up to $11 \%$ of all women after pelvic lym- 


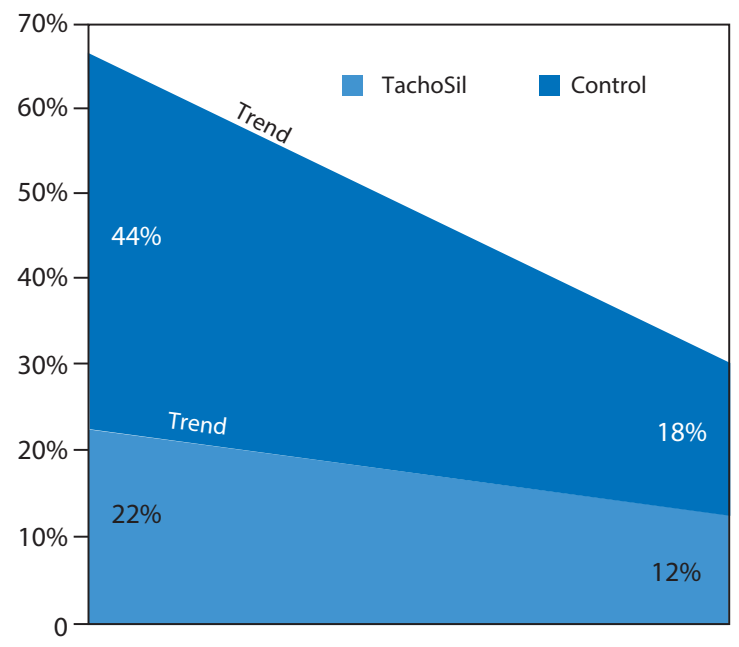

Figure 4. Incidence of lymphocele (\%) in the side in which TachoSil was applied versus that in the control after completion of adjuvant therapy

phadenectomy $[1,11]$, but in only $2 \%$ of all women who had symptomatic lymphocele and required a therapeutic intervention [11]. Tinelli et al. [8], showed that in the development of asymptomatic lymphoceles, in a comparison of 30 women in whom TachoSil had been applied versus 28 women in whom TachoSil had not been applied, a significant difference existed [7 (23.3\%) vs 9 (57.7\%), respectively] ( $p<0.05)$. This research group, however, found no significant differences between the two groups in the occurrence of symptomatic lymphoceles and associated surgeries [1]. In contrast, in a 60-month follow-up study done by Radosa et al. [12] of 102 symptomatic lymphocele patients, in whom laparoscopy was initially performed, laparoscopy was reported to be less recurrent when TachoSil was used.

Tinelli et al. [1], conducted two randomized controlled studies assessing the effect of a collagen patch covered with human coagulation factors (TachoSil) as a lymphocele preventative after pelvic lymphadenectomy was performed in women with endometrial cancer. The first study involved 58 patients with diagnosed endometrial cancer subjected to hysterectomy and pelvic lymphadenectomy. The first group consisted of 30 patients treated with a standard technique followed by the use of TachoSil. The remaining patients were treated using standard technique without the use of TachoSil (control). Operational parameters were collected, and ultrasound scans were performed on days 7, 14 and 28 post-surgery to assess the presence and development of both symptomatic and asymptomatic lymphocele, as well as to ascertain the need for further surgical intervention. The time and volume of drainage was also established. In both groups, the same number of lymph nodes was removed. It was shown that in the first group, the volume of drainage was smaller, in addition, lymphocele developed in seven patients from the TachoSil Group and in 16 patients from the control group. In the TachoSil Group, only three lymphoceles were symptomatic versus nine symptomatic in the control group. This represented statistically significant differences. What is more, percutaneous drainage was performed in one case in the first group and in four cases in the second group. It was concluded that the intraoperative use of TachoSil reduces the incidence of postoperative lymphocele after pelvic lymphadenectomy and may be considered as an additional therapeutic option in reducing the volume of drainage and preventing lymphocele formation [8].

In the second study conducted by Tinelli et al. [1], the same postoperative parameters were evaluated via ultrasound examination in a scheme analogous to that used in the previous study. In the first group, 26 patients underwent pelvic lymphadenectomy and TachoSil was applied, in the second group, the control consisting of 29 people, the procedure was performed without collagen patch application. In this scenario, it was shown that intra-operative laparoscopic application of the TachoSil seemed to reduce the rate of incidence of lymphocele formation after pelvic lymphadenectomy and provided an additional treatment option in reducing the volume of drainage and preventing the development of lymphocele.

The method used in the above study conducted by Tinelli et al. [1], was used in the surgery protocol of the study carried out in our center, with the outcome as described in the Results section. Both the difference in prevalence and the volume of lymphocele after using TachoSil after 30 days from surgery turned out to be statistically significant (44\% in the control group versus $22 \%$ in the TachoSil Group). In the group of patients on the side of which TachoSil was not used, symptomatic cysts tended to form.

We then re-evaluated the clinical situation after the complementary oncological treatment. On doing so, we observed a decreased tendency of occurrence of lymphocele following post-adjuvant therapy in the TachoSil Group. Indeed, when compared to the control group (without TachoSil application), $12 \%$ of the TachoSil Group contracted lymphocele, versus $18 \%$ of the control group (without TachoSil). In addition, while Simonato et al. [13], and Tinelli et al. [1], claim that the application of a collagen-fibrin patch $\left(\right.$ TachoSil $\left.^{\circledR}\right)$ reduced the number of symptomatic pelvic lymphoceles by at least $66 \%$ after 30 days of surgery $[1,8,13]$, in our study, TachoSil decreased lymphocele by about $56 \%$ after 30 days of intervention and $88 \%$ after completion of adjuvant therapy.

\section{CONCLUSIONS}

TachoSil is a useful support treatment option for reducing drainage volume and preventing lymphocele development after lymphadenectomy. 


\section{Funding}

Funding was supported through a Takeda scientific grant.

\section{Availability of data and materials}

The datasets used and/or analyzed during the current study are available from the corresponding author on reasonable request.

\section{Authors' contributions}

I.W - acquisition and interpretation of data, writing the manuscript, statistical analysis; A.W - conception and study design, performing the USG scan, writing the manuscript; K.S - interpretation of data; writing the manuscript, collection of data; T.R - conception and study design, collection of data, writing the manuscript, giving final approval of the version to be published, P.M — conception and study design, writing the manuscript. All authors read and approved the final manuscript.

\section{Ethics approval and consent to participate and for publication}

The study was approved by the Bioethics Committee of the Medical University of Lublin, Lublin, Poland (KE0254/276/2013) in accordance with The Code of Ethics of the Declaration of Helsinki. Informed consent was obtained from all individual participants included in the study.

\section{Conflict of interest}

We declare that we have no conflict of interest.

Takeda provided TachoSil used in this study.

\section{REFERENCES}

1. Tinelli A, Mynbaev OA, Tsin DA, et al. Lymphocele prevention after pelvic laparoscopic lymphadenectomy by a collagen patch coated with human coagulation factors: a matched case-control study. Int J Gynecol Cancer. 2013; 23(5): 956-963, doi: 10.1097/IGC.0b013e31828eeea4, indexed in Pubmed: 23574881.
2. Frost JA, Webster KE, Bryant A, et al. Lymphadenectomy for the management of endometrial cancer. Cochrane Database Syst Rev. 2015(9): CD007585, doi: 10.1002/14651858.CD007585.pub3, indexed in Pubmed: 26387863.

3. Achouri A, Huchon C, Bats AS, et al. Postoperative lymphocysts after lymphadenectomy for gynaecological malignancies: preventive techniques and prospects. Eur J Obstet Gynecol Reprod Biol. 2012; 161(2): 125-129, doi: 10.1016/j.ejogrb.2011.12.021, indexed in Pubmed: 22364898.

4. Spotnitz WD, Burks S. Hemostats, sealants, and adhesives: components of the surgical toolbox. Transfusion. 2008; 48(7): 1502-1516, doi: 10.1111/j.1537-2995.2008.01703.x, indexed in Pubmed: 18422855.

5. Buda A, Ghelardi A, Fruscio R, et al. The contribution of a collagen-fibrin patch (TachoSil) to prevent the postoperative lymphatic complications after groin lymphadenectomy: a double institution observational study. Eur J Obstet Gynecol Reprod Biol. 2016; 197: 156-158, doi: 10.1016/j. ejogrb.2015.12.005, indexed in Pubmed: 26765122.

6. Grimm C, Polterauer S, Helmy-Bader S, et al. A collagen-fibrin patch $\left(\right.$ TachoSil $\left.{ }^{\oplus}\right)$ for the prevention of symptomatic lymphoceles after pelvic lymphadenectomy in women with gynecologic malignancies: a randomized clinical trial. BMC Cancer. 2014; 14(1):635-145, doi: 10.1186/14712407-14-635, indexed in Pubmed: 25175029.

7. Mining L, Patrono MG, Cárdenas-Rebollo JM, et al. Use of TachoSil ${ }^{\circledR}$ to Prevent Symptomatic Lymphocele after an Aggressive Tumor Debulking with Lymphadenectomy for Advanced Stage Ovarian Cancer. A Pilot Study. Gynecol Obstet Invest. 2016; 81(6):497-503, doi: 10.1159/000443640, indexed in Pubmed: 27046053.

8. Tinelli A, Giorda G, Manca C, et al. Prevention of lymphocele in female pelvic lymphadenectomy by a collagen patch coated with the human coagulation factors: a pilot study. J Surg Oncol. 2012; 105(8): 835-840, doi: 10.1002/jso.22110, indexed in Pubmed: 21987409.

9. Song SY, Park M, Kang BH, et al. Distribution of lymphocele following lymphadenectomy in patients with gynecological malignancies. Obstet Gynecol Sci. 2020; 63(6): 700-708, doi: 10.5468/ogs.20110, indexed in Pubmed: 32814372

10. Achouri A, Huchon C, Bats AS, et al. Complications of lymphadenectomy for gynecologic cancer. Eur J Surg Oncol. 2013; 39(1): 81-86, doi: 10.1016/j.ejso.2012.10.011, indexed in Pubmed: 23117018.

11. $\mathrm{Xu} \mathrm{H}, \mathrm{Chen} \mathrm{Y,} \mathrm{Li} \mathrm{Y,} \mathrm{et} \mathrm{al.} \mathrm{Complications} \mathrm{of} \mathrm{laparoscopic} \mathrm{radical} \mathrm{hysterec}$ tomy and lymphadenectomy for invasive cervical cancer: experience based on 317 procedures. Surg Endosc. 2007; 21(6): 960-964, doi: 10.1007/s00464-006-9129-0, indexed in Pubmed: 17287919.

12. Radosa MP, Diebolder H, Camara O, et al. Laparoscopic lymphocele fenestration in gynaecological cancer patients after retroperitoneal lymph node dissection as a first-line treatment option. BJOG. 2013; 120(5): 628-636, doi: 10.1111/1471-0528.12103, indexed in Pubmed: 23320834

13. Simonato $A$, Varca $V$, Esposito $M$, et al. The use of a surgical patch in the prevention of lymphoceles after extraperitoneal pelvic lymphadenectomy for prostate cancer: a randomized prospective pilot study. J Urol. 2009; 182(5): 2285-2290, doi: 10.1016/j.juro.2009.07.033, indexed in Pubmed: 19762048 